\title{
A user interface for mobile robotized tele-echography
}

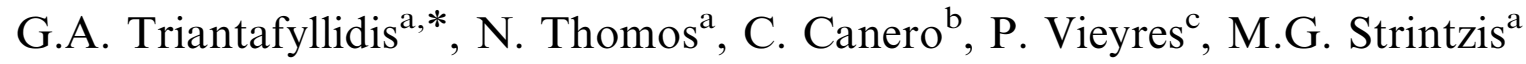 \\ ${ }^{a}$ Informatics and Telematics Institute ITI-CERTH, Thessaloniki, Greece \\ ${ }^{\mathrm{b}}$ Computer Vision Center, UAB, Barcelona, Spain \\ ${ }^{\mathrm{c}}$ Laboratoire Vision and Robotique Universite d'Orleans, Bourges, France
}

Available online 12 September 2006

\begin{abstract}
Ultrasound imaging allows the evaluation of the degree of emergency of a patient. However, in many situations no experienced sonographer is available to perform such echography. To cope with this issue, the OTELO project "mObile Tele-Echography using an ultra-Light rObot" (OTELO) aims to develop a fully integrated end-to-end mobile tele-echography system using an ultralight, remotely controlled six degree-of-freedom (DOF) robot. In this context, this paper deals with the user interface environment of the OTELO system, composed by the following parts: an ultrasound video transmission system providing real-time images of the scanned area at each moment, an audio/video conference to communicate with the paramedical assistant and the patient, and finally a virtual reality environment, providing visual and haptic feedback to the expert, while capturing the expert's hand movements with a one-DOF hand free input device.
\end{abstract}

(C) 2006 Elsevier B.V. All rights reserved.

PACS: 07.05.Wr; 84.40.Va; 87.57.Ra

Keywords: Tele-echography; User interface; Virtual reality environment

\section{Introduction}

Mobile interconnectivity and telemedicine are important issues for achieving effectiveness in health care, since medical information can be transmitted faster and physicians can make diagnoses and treatment decisions faster $[1,2]$. Moreover, there is the need to perform quick and reliable diagnosis to evaluate the gravity of the pathology in presence of clinical symptoms. Many pathological situations (abnormal heart rate, pericardia collection, mitral prolaps, cholecystis, renal lithiasis, normal and ectopic pregnancies, ovarian cyst, acute appendicitis, phlebitis...) may occur at any time. Ultrasound echography and Doppler are noninvasive methods easy to use and very well adapted for routine examination such as for hospital. In this context, the "mObile Tele-Echography using an ultra-Light rObot" (OTELO) system proposes a fully integrated end-to-end mobile tele-echography system

\footnotetext{
${ }^{*}$ Corresponding author.

E-mail address: gatrian@iti.gr (G.A. Triantafyllidis).
}

for population groups that are either temporarily or permanently not served locally by medical experts.

An important issue is that the current common ultrasound examination techniques require on-site specialized operators, who are not always available in many health centers. For this reason, OTELO is designed to enable a remote examination by means of a portable ultrasound probe holder robotic system, in conjunction with new mobile communications technologies. The system reproduces the expert's hand movements during the ultrasound examination, while transmitting in real-time ultrasound images to the expert site, where force feedback control is combined with virtual reality for the rendering of the distant environment. Hence, just a paramedical assistant is required on the patient site.

In this paper, the proposed user interface for the OTELO system is presented. Section 2 briefly describes the OTELO system and its components, while in Sections 3 and 4 the virtual reality environment and the graphical user interface design and implementation are elaborated and discussed. Special care must be taken when designing the user 
interface for making it as user-friendly as possible. With this aim, all the final design decisions have been validated by medical experts and motivated by the clinical tests that have been carried out.

\section{Overall description of the system}

The main drawback of the ultrasound technique is that the quality of the examination highly depends on the operator's skills. The technique is operator-dependant: a few degrees modification of the ultrasound probe orientation on the patient's skin could lead to miss or loose the investigated organ.

In response to users specifications, the chosen kinematics structure, proposed by the OTELO system, is a light weight and portable six degree-of-freedom (DOF) mechanical system [3] that enables the manipulation of the ultrasound probe without displacement of the contact point on the skin.

Three main parts can be identified for the OTELO teleechography chain: (a) The expert station where the ultrasound expert receives, with a user-friendly interface and in almost real-time, ultrasound images of the patient's organ. The expert based on these images can modify the orientation of the remote real ultrasound probe by changing the position of the input device. (b) The patient station is equipped with the six-DOF robotic probe holder system and an ultrasound device. It receives the positioning data from the input device localization sensor, and reproduces the expert's hand movements. (c) Two kinds of communication links between the two stations are supported: (i) terrestrial when ISDN infrastructure is available, (ii) satellite (mobile or fixed), when coverage is possible, in order to reach isolated populations Moreover, a videoconference link between the two stations offers a closer medical and technical relationship between the expert, the paramedic and the patient.

At the beginning of the tele-echography act, a prepositioning of the robotic system on the patient's skin has to be performed by the paramedical assistant. This positioning is very important since it conditions the overall diagnosis. During the tele-echography, the paramedic maintains the robot on the patient's skin. Therefore, for the tele-echography act, which involves an ultrasound specialist and a paramedical assistant dealing with complex mechatronic tele-operated structures, a user-friendly interface must be provided. This will help the paramedic to correctly position the robot, and the expert to better control the robot and to follow its positioning. The paramedic assistant, who continuously maintains the robot during the tele-examination, may be asked to move it on the patient's body according to the expert information.

\section{Virtual reality environment}

In order to increase the sensation of tele-presence, a virtual reality environment has been developed. This environment renders the patient body, the ultrasound probe and optionally the robot, allowing the expert to get a better insight of where the ultrasound probe is placed (with no use of a special stereoscopic display). The patient model is selected to fit the patient's body characteristics, prior to the examination at the patient station among a $3 \mathrm{D}$ mesh library.

Regarding the input device for the expert hand movements, two options are available: one of the options is to use a PHANToM ${ }^{\mathrm{TM}}$ device, which is able to provide force feedback and hence enables the expert to feel the pressure exerted on the patient skin. The other option is the utilization of a patented, one-DOF hand-free device [4], an input device that has been specially developed for the OTELO system and that provides information about the expert movements [5]. Hence, using the proposed virtual environment the expert can perform a remote examination as he/she is virtually next to the patient.

Note that the above can be achieved, provided that an accurate knowledge of the actual robot position and orientation onto the patient skin is available. To cope with this issue, the chosen approach allows the expert to select the best 3D model for the patient's body from an available $3 \mathrm{D}$ model library and then determine the desired position and orientation of the robot onto the patient body using the virtual reality environment. This information is sent to the patient station, where it is rendered. Therefore, the paramedical assistant has a good understanding of the desired positioning of the robot. Besides, the expert is provided with the patient ambient video to confirm it. Once the robot is correctly placed, the expert gains control of the robot and starts the examination using either the PHAN$\mathrm{ToM}^{\mathrm{TM}}$ device or the one-DOF hand-free device.

\subsection{Selecting the robot positioning}

Defining a point in $3 \mathrm{D}$ coordinates is usually a difficult task for inexperienced users, especially if no specialized input devices are available. However, when selecting a positioning for the robot, two restrictions are held: Firstly, that the robot must be in contact with the patient skin, and hence the position is restricted to the surface representing the patient. Secondly, the orientation of the robot vertical axis should be perpendicular to the patient skin at the contact position of the robot. According to these restrictions, a user-friendly interface for determining the target robot positioning has been designed.

To select the position of the robot, the expert clicks on the image displaying the patient model. The 3D target robot position is, therefore, obtained by intersecting the corresponding projective ray (defined as the line from the clicked position on the rendered image to the camera eye) with the patient model. Then, the orientation of the vertical axis of the robot is set to the normal of the patient model surface at the intersecting point. Both the robot and the patient can then be rendered to the expert, and eventually 
the expert can refine the obtained robot orientation by rotating the robot among the vertical axis using the mouse.

\subsection{Robot control}

As mentioned above, the position and orientation of the probe is sent to the patient station, where it is reproduced by the robot. However, tele-robotic applications where the distance between the master and slave stations is large (such as in the OTELO tele-echography case) are operating with large communication delays. Actually, in the tests carried out with the first OTELO prototype, one of the main complaints of the medical experts was the problems introduced by the delay between the movement of the input device and the actual movement of the robot at the Patient Station. These problems were alleviated by the experts by moving the one-DOF hand-free device slowly, allowing the robot to follow the target position. However, the expert had no feedback about the actual probe movement.

Two possible solutions have been proposed to provide the expert with such feedback: a visual-feedback and a force-feedback approach. In both the cases, the current position and orientation of the ultrasound probe is sent to the Expert Station. In the visual-feedback approach, the target position of the ultrasound probe is rendered as a red wireframe, and the real one as a solid, realistic model. This helps the expert to understand whether the input device is being moved too fast, and where the probe really is at each moment. On the other side, the force-feedback consists of a new added force effect to the haptic environment that attracts the virtual probe to the current position of the ultrasound probe. Thus, whenever the expert moves the probe too fast, he/she will experience a resistance against the movement. This approach could only be used with the PHANToM device. Both techniques can be combined to assist the expert.

\section{Graphical user interface}

After being ready for the remote examination, the doctor can switch between the robot positioning and robot control.

\subsection{Robot positioning}

Fig. 1 illustrates the window displayed during the robot positioning procedure.

A virtual environment enables the expert to select the desired position and orientation of the robot by a single mouse click. It is worth noting that the paramedical assistant monitors the target robot location while holding the robot, and hence cannot interact with the virtual environment to select a proper projection of the scene. For this reason, the information relating the point of view at the Expert Station is sent to the Patient Station, in order to generate both renderings from the same point of view. Additionally, a high quality (CIF size) ambient video is

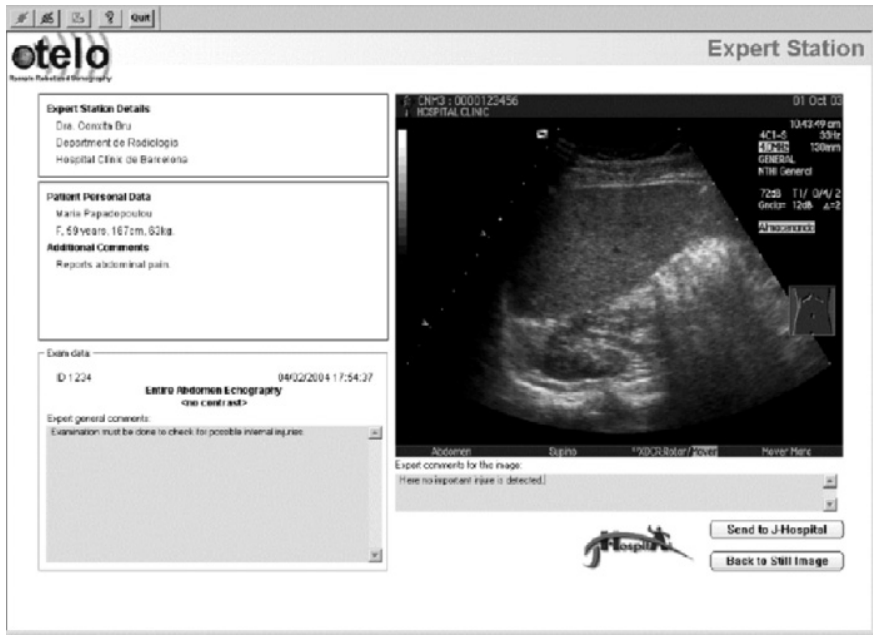

Fig. 1. Displayed window at the expert station during robot positioning.

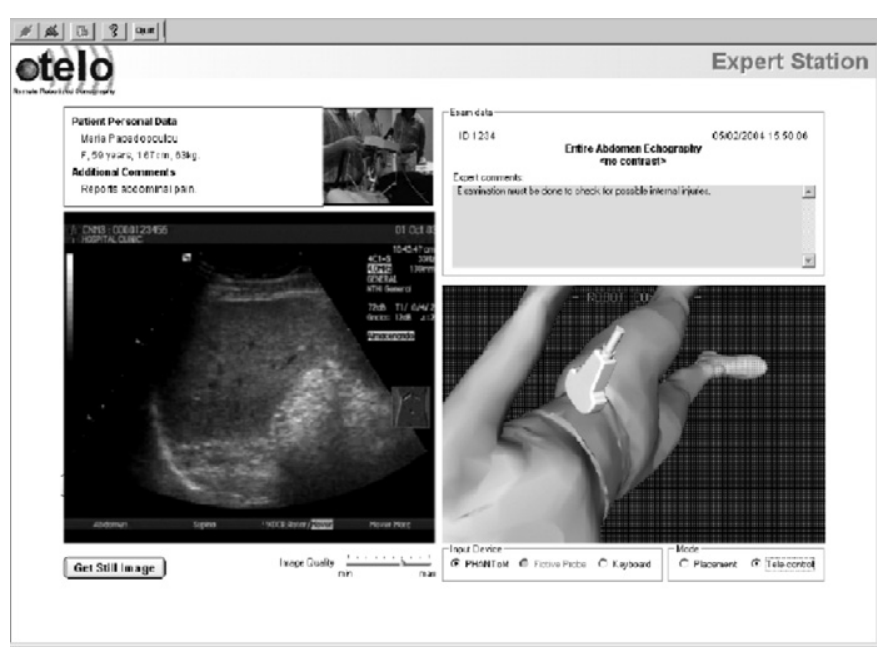

Fig. 2. Displayed window at the expert station during robot control.

transmitted, allowing the expert to monitor the robot positioning. When the robot is correctly positioned, the expert can gain control of the robot by pressing on the "Robot Control" radio button.

\subsection{Robot control}

Fig. 2 presents the window proposed for robot control. The input device tracks the position and orientation coordinates of the expert's hand movements, which are sent to the patient station to be reproduced by the robot. The virtual environment renders the patient body and the ultrasound probe as described in Section 3.2. Besides, the expert receives CIF size ultrasound images from the patient station along with QCIF ambient video. Finally, whenever the expert feels that he/she should have a still image of a better quality, he/she can use the "Get Still Image" button, which opens a window with the full quality image, allowing to store it into a medical database. 


\section{Medical database interface}

Prior to an echographic act, the physician interviews the subject and needs to access eventually his/her medical files. This information can be a common base for scientific statistical analysis for medicine and pharmacology. The underlying idea is, therefore, that medical and paramedical personnel store and retrieve relevant medical data of the patient with any institute joining the system. Moreover, it also provides the availability for queries of a wider set of medical cases, which can be very useful in determining decisions about a new patient taking into account similar cases.

Being aware of the advantages of such a database facility, the OTELO system has been designed to include an interface that allows the connection with the J-Hospital medical database system [6], which provides a common base of multimedia data relevant to any patient in any institution connected to the network. When the physician decides to store a full-quality image into the database, he/ she presses the "J-Hospital Interface" button. A new interface is launched (see Fig. 3), showing the patient medical data and the report written by the expert until the moment.

\section{Conclusions}

A new implementation for the OTELO tele-echography system user interface is proposed. It allows the expert and his remote paramedical assistant to efficiently set and control the positioning of the robotic system prior to the tele-echography act. Thanks to the video/audio conference links, specifically developed for the system, the ultrasound specialist is always in contact with the patient for his/her comfort and well-being. The paramedic has full control of the robotic system in case of emergency and can always, thanks to the user interface, discuss the situation with the distant expert. Although a study of the different specific scenarios for the ultrasound image and robotic control communication depending on the available bandwidth has been already carried out (see Ref. [7] for more details), the measures usually used to evaluate image quality cannot provide enough information about the diagnostic usability of the whole system. To do such an evaluation, clinical tests involving medical expert sonographers and real patients is a must. The next foreseen step is therefore to do an extensive clinical test of the whole system for different bandwidth scenarios and to evaluate the best configuration for each, as far as the minimum bandwidth requirements to assure usability.

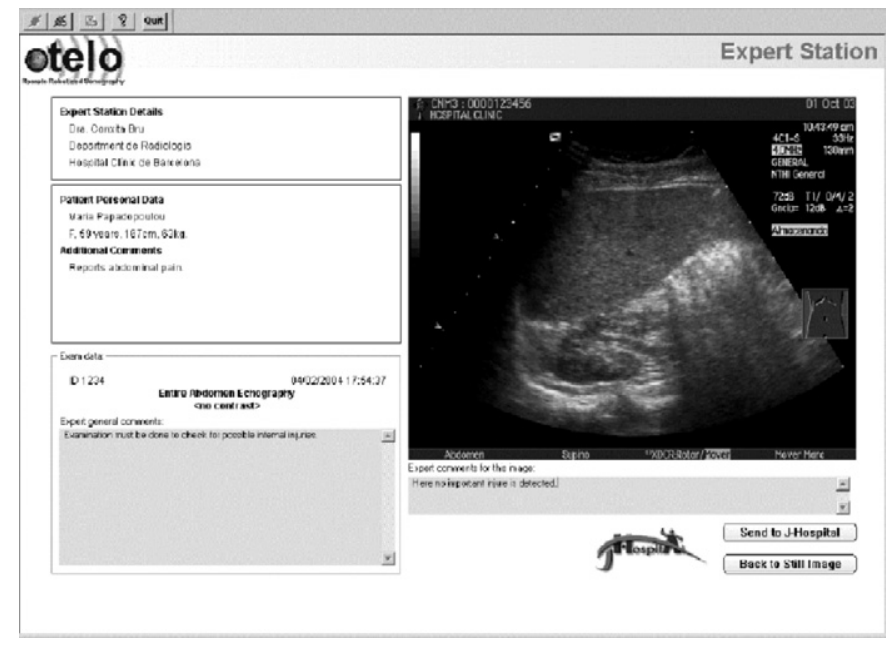

Fig. 3. Medical database interface.

\section{Acknowledgments}

This work was supported by the EU project IST-200132516 OTELO [8]. The authors would like to thank the partners in OTELO project for their support.

\section{References}

[1] D. de Cunha, P. Gravez, C. Leroy, E. Maillard, J. Jouan, P. Varley, M. Jones, M. Halliwell, D. Hawkes, P.N.T. Wells, L. Angelini, The MIDSTEP system for ultrasound guided remote telesurgery, in: 20th Annual Intelligent Conference, IEEE Engineering in Medicine and Biology Society, 1998, pp. 1266-1269.

[2] G. Kontaxakis, S. Walter, G. Sakas, EU-TeleInViVo: an integrated portable telemedicine workstation featuring acquisition, processing and transmission over low-bandwidth lines of 3D ultrasound volume images, in: Proceedings of IEEE EMBS ITAB 2000, Arlington, VA, November 2000, pp. 158-163.

[3] L. Al-Bassit, G. Poisson, P. Vieyres, Kinematics of a 6DOF robot for tele- echography, in: 11th International Conference on Advanced Robotics (ICAR 2003), 2003.

[4] G. Poisson, P. Vieyres, F. Courreges, Sonde Fictive Echographique National Patent n0201027, Extended to European Patent n03290168.8, Orleans University, 2002-2003.

[5] N. Smith-Guerin, L. Al-Bassit, F. Courreges, G. Poisson, C. Delgorge, P. Arbeille, P. Vieyres, Clinical validation of a mobile patient-expert tele-echography system using ISDN lines., in: Proceedings of the Fourth Annual IEEE/EMBS ITAB'03, 2003.

[6] KELL, J-Hospital Product Presentation. (Available at http://www. kell.it).

[7] G.A. Triantafyllidis, N. Thomos, G. Nikolakis, D. Tzovaras, G. Litos, M.G. Strintzis, in: R. Istepanian, S. Laxminarayan, C.S. Pattichis, (Eds.), User Interface enviroment and Image Communication in Mobile Tele-Echography, M-Health: Emerging Mobile Health Systems, Series: International Topics in Biomedical Engineering, Springer, Berlin, ISBN: 0-387-26558-9, 2006.

[8] OTELO, mObile Tele-Echography using an ultra-Light rObot, www.bourges.univ-orleans.fr/otelo/home.htm 